\title{
Taxa de fertilidade de novilhas de diferentes grupos genéticos com primeiro serviço aos 14 meses de idade
}

[Fertility rate of heifers of different genetic groups with 1st service at 14 months of age]

\author{
N.V. Sollecito ${ }^{1}$, V.J. Andrade ${ }^{2}$, F.A. Barbosa ${ }^{2}$,C.F. Lobo ${ }^{1}$, \\ H.O. Azevedo ${ }^{1}$, P.H.S. Guimaraes ${ }^{3}$
}

${ }^{1}$ Aluno de pós-graduação - Escola de Veterinária - UFMG - Belo Horizonte, MG

${ }^{2}$ Escola de Veterinária - Universidade Federal de Minas Gerais - UFMG - Belo Horizonte, MG

${ }^{3}$ ICAL Fazenda do Tronco

\section{RESUMO}

Foram avaliadas as taxas de reconcepção de 98 novilhas primíparas (34 Guzerá - G, 32, 1/2 Guzerá x 1/2 Nelore - GN e 32, $1 / 2$ Red Angus x 1/2 Nelore - AN) com 14 meses de idade e peso médio de aproximadamente $249,65 \mathrm{~kg}$, criadas em pastos de Brachiaria brizantha cv Marandu. Na segunda estação de monta (EM), foram utilizadas apenas as 36 primíparas gestantes na primeira EM (três G, nove GN e 24 $\mathrm{AN})$, com média de idade de 26 meses e peso corporal de $313,67 \pm 25,01 \mathrm{~kg}, 336,50 \pm 45,84 \mathrm{~kg}$ e $399,86 \pm 44,45 \mathrm{~kg}$, respectivamente, para as fêmeas dos grupos G, GN e AN. O grupo AN apresentou ganho médio diário $(\mathrm{GMD})$ de $0,30 \pm 0,06(\mathrm{~kg} / \mathrm{dia})$ e maiores taxas $(58,33)$ de reconcepção $(\mathrm{P}<0,05)$, comprovando que a heterose resultante do cruzamento entre raças distintas com maior distância genética (Bos taurus taurus x Bos taurus indicus) proporciona maior desempenho produtivo e reprodutivo.

Palavras-chave: grupos genéticos, primíparas, taxa de reconcepção, heterose

\section{ABSTRACT}

A total of 98 heifers of three genetic groups: 34 Guzerá (G), 32, 1/2 Guzerá x 1/2 Nelore (GN) and 32 1/2 Red Angus $x$ 1/2 Nelore (AN), 14 month old and average body weight of 249,65kg, raised in Brachiaria brizantha $c v$ Marandu were used to evaluate reconception rates. In the second breeding season (BS), only those 36 pregnant in the first BS (three G, nine GN and 24 AN), age 26 months and body weight of $313.67 \pm 25.01 \mathrm{~kg}, 336.50 \pm 45.84 \mathrm{~kg}$ and $399.86 \pm 44.45 \mathrm{~kg}$, respectively, $G, G N$ and $A N$ were evaluated. The highest reconception rate $(58.33 \%, P<0.05)$ was registered for the AN group and showed that average daily gain of $0.30 \pm 0.06 \mathrm{~kg}$, showing that crosses between Bos taurus taurus $\mathrm{x}$ Bos taurus indicus leads to higher reproductive and productive performances.

Keywords: genetic group, heifers, reconception rate, heterosis

\section{INTRODUÇÃO}

A pecuária de corte no Brasil é uma atividade cada vez mais pressionada pela sociedade e pelos mercados, interno e externo, os quais exigem maior produtividade do setor e maior qualidade do produto produzido.

O aprimoramento do sistema produtivo deve ser atingindo dando-se atenção especial ao setor reprodutivo. A eficiência reprodutiva pode ser considerada como uma das mais importantes características econômicas dentro do sistema produtivo, devendo merecer maior atenção dos criadores (Fonseca, 1991).

Ao lado da precocidade sexual, outro índice marcante na reprodução do rebanho e que determina o potencial de produtividade dele é a taxa de reconcepção das fêmeas, que corresponde à capacidade de gerar novamente um produto na estação de monta subsequente.

Recebido em 11 de setembro de 2014

Aceito em 26 de novembro de 2015

E-mail: nat.sol86@yahoo.com.br 
A taxa de reconcepção está associada com os ganhos médios de peso entre a desmama ao sobreano e a maturidade (Silva et al., 2010) e com o monitoramento do escore de condição corporal (ECC), que é indispensável para que as fêmeas voltem a ciclar e repetir a prenhez na segunda estação de monta (Richard et al.,1986).

Quando as condições ambientais apresentam restrições para o desenvolvimento normal, as novilhas manejadas em condições idênticas podem apresentar desempenhos diferentes, já que, além da nutrição, outra causa relevante para o sucesso reprodutivo está associada ao seu genótipo (Pereira Neto e Lobato, 1998; Paim 2010). A utilização de cruzamentos pode ser uma ferramenta para aumentar o grau de heterose e da seleção de animais mais aptos para alcançar elevadas taxas de reconcepção, possibilitando um aumento nos índices produtivos e reprodutivos.

Diante dos muitos desafios e obstáculos pelos quais a pecuária de corte vem passando, o uso de um plano alimentar compatível com as demandas de cada categoria animal deve ser previamente disponibilizado (Rovira, 1996) concomitantemente ao uso do cruzamento entre raças ou entre raças ou subespécie (Bos taurus taurus $x$ Bos taurus indicus), promovendo a possibilidade de se aproveitarem as características favoráveis de cada uma delas, juntamente com o maior desempenho ponderal provocado pela heterose (Alencar, 2004).

Objetivou-se com esse estudo avaliar as taxas de reconcepção das primíparas de três diferentes grupos genéticos em pastos de Brachiaria brizantha cv. Marandu.

\section{MATERIAL E MÉTODOS}

O experimento foi realizado após a aprovação do Comitê de Ética em Experimentação AnimalUFMG, reconhecido pelo número de protocolo 116/11, na Fazenda do Tronco, localizada no município de Felixlândia, estado de Minas Gerais, com precipitação pluviométrica anual média de $1.235 \mathrm{~mm}$. O período experimental foi de 9 de fevereiro de 2012 a 18 de junho de 2013.

A área experimental era composta por seis piquetes estabelecidos com a gramínea Brachiaria brizantha cultivar Marandu, com área de 15 ha cada, totalizando 90 ha de pastagem.
Todos os piquetes eram providos de bebedouro e cochos cobertos para fornecimento de suplementação mineral e/ou proteinado.

$\mathrm{Na}$ primeira estação de monta (EM), foram trabalhadas ao todo 98 novilhas, sendo 34 fêmeas da raça Guzerá $(\mathrm{G}), 32$ 1/2 Guzerá x 1/2 Nelore (GN) e 32 1 $1 / 2$ Red Angus x 1/2 Nelore (AN), com idade média de 14 meses. Na segunda estação de monta, foram utilizados apenas os animais positivos para prenhez na primeira estação de monta, equivalendo a um total de 36 novilhas, sendo três da raça Guzerá $(\mathrm{G})$, nove $1 / 2$ Guzerá $1 / 2$ Nelore (GN) e 24 1/2 Red Angus 1/2 Nelore (NA), com idade média de 26 meses e peso vivo médio inicial de $313,67 \pm 25,01 \mathrm{~kg}$ para $\mathrm{G}$, $336,50 \pm 45,84 \mathrm{~kg}$ para $\mathrm{GN}$ e $399,86 \pm 44,45 \mathrm{~kg}$ para AN. Todos os animais eram originários de um mesmo rebanho e foram mantidos sob as mesmas condições de ambiente, manejo e alimentação. Receberam tratamentos contra carrapatos, moscas e as devidas vacinações preventivas.

Durante a segunda EM, as 36 novilhas foram manejadas num único lote, utilizando-se o sistema de pastejo rotacionado, com o rodízio entre os piquetes sendo realizado a cada 14 dias. A taxa de lotação média durante o experimento foi de $0,5 \mathrm{UA}(450 \mathrm{~kg} \mathrm{PV}) / \mathrm{ha}$.

A primeira EM teve início em $1^{\circ}$ de fevereiro de 2012 e terminou em $1^{\circ}$ de maio do mesmo ano. Ao início da EM, foi utilizado um protocolo hormonal e inseminação artificial em tempo fixo (IATF), com repasse com touro na relação de 1:30 (touro:vaca) até o encerramento da estação de monta. $O$ diagnóstico de gestação foi realizado por palpação retal e confirmação por ultrassonografia transretal após 30 dias do fim da EM.

A segunda estação de monta teve duração de 90 dias, com início em $1^{\circ}$ de janeiro de 2013 e término em $1^{\circ}$ de abril do mesmo ano, com participação apenas daquelas novilhas com diagnóstico de gestação positivo na primeira estação reprodutiva. $\mathrm{Na}$ segunda estação de monta, foi utilizada monta natural com diagnóstico de gestação, por palpação retal, efetuado 60 dias após o fím dela.

A amostragem das pastagens foi feita a partir de cortes em 10 áreas/piquetes determinadas por um quadrado metálico de 1x1m, escolhidas 
aleatoriamente na entrada e saída dos animais, com cortes da forragem rente ao solo, conforme McMeniman (1997). Após a pesagem do material coletado, foi efetuada a homogeneização de idênticas amostras e retiradas duas subamostras compostas, uma para análise de disponibilidade total e outra para determinação do percentual das frações matéria seca verde (MSV), matéria seca morta (MSM).

Amostras da pastagem selecionada pelo animal foram coletadas pela técnica do pastejo simulado segundo Euclides et al. (1998). As amostras foram secas em estufa a $65^{\circ} \mathrm{C}$, foi calculada a produção em $\mathrm{kg} / \mathrm{ha}$ de MS, e, depois, elas foram encaminhadas para análises bromatológicas, determinando-se os teores de proteína bruta (PB), nutrientes digestíveis totais (NDT) e digestibilidade in vitro da matéria seca (DIVMS) de acordo com a metodologia descrita por Detmann et al. (2012).

As pesagens dos animais foram realizadas no início do experimento e a cada 56 dias, conjuntamente à avaliação do escore de condição corporal de cada animal numa escala de um a nove, segundo Lowman et al. (1976). Os pesos e os ganhos de pesos foram obtidos sem jejum prévio de 12 horas. O ganho de peso médio diário (GMD) foi obtido pela diferença entre peso final e inicial dos animais em cada período experimental, dividido pelo número de dias do período.

As variações diárias de peso na segunda estação de monta foram calculadas como a diferença entre o peso no final e no início dela, dividida pelo número de dias entre as pesagens, conforme Hight (1966).

Foi utilizado o delineamento experimental inteiramente ao acaso com parcelas subdivididas no tempo, considerando os animais como repetições. Os dados referentes ao peso, ganho de peso e escore de condição corporal foram submetidos à análise de variância e ao teste $\mathrm{F}$, utilizando-se o seguinte modelo matemático na análise:

$Y i j k l=\mu+T i+A j(T) i+P k+\left(T^{*} P\right) i k+\varepsilon i j k$, em que:

Yijk = variáveis dependentes;

$\mu=$ média de todas as observações;

$\mathrm{Ti}=$ efeito do i-ésimo grupo racial;
Aj $(\mathrm{T}) \mathrm{i}=$ efeito do j-ésimo animal dentro do iésimo grupo racial (erro $\mathrm{A}$ );

$\mathrm{Pk}=$ efeito do k-ésimo período; $\left(\mathrm{T}^{*} \mathrm{P}\right) \mathrm{ik}=$ efeito de interação entre o i-ésimo grupo racial e o k-ésimo período;

$\varepsilon i j \mathrm{k}=$ erro residual (erro B).

Quando detectada diferença entre os grupos genéticos, foi realizado o teste de Tukey para comparação de médias nas análises da variação de peso. Já o escore de condição corporal foi analisado pelo teste não paramétrico de KruskalWallis. As análises estatísticas para as variáveis peso e condição corporal foram realizadas usando-se o procedimento GLM do pacote computacional SAS (2004).

Os resultados do diagnóstico de gestação para taxa de reconcepção dos diferentes grupos genéticos foram realizados pelo teste do quiquadrado de independência $\left(\chi^{2}\right)$ (Snedecor e Cochran, 1980), utilizando-se o programa estatístico SAS, versão 6.08 (SAS (2004), com nível máximo de $5 \%$ de significância.

\section{RESULTADO E DISCUSSÃO}

Durante todo o período experimental, a produção de matéria seca total (MST) foi adequada, apresentando valores acima de $2.000 \mathrm{~kg} / \mathrm{ha}$ conforme preconizado por Flores et al. (2008). Os valores médios de produção de matéria seca verde (MSV) de 793,8kg/ha em agosto e de $997,0 \mathrm{~kg} / \mathrm{ha}$ em outubro de 2012 apresentaram-se como possíveis limitantes, com valores de produção menores que o limite preconizado por Euclides (2000) de $1.000 \mathrm{~kg}$ de MVS/ha.

Nas análises da composição químicobromatológica da amostra de pastejo simulado coletada durante $\mathrm{o}$ experimento, foram encontrados valores de PB de 1,5\% a 13,9\%, NDT de $29,14 \%$ a $64,9 \%$ e digestibilidade in vitro da matéria seca (DIVMS) de 30,7\% a $64,7 \%$, que sofreram variações conforme a época do ano e a idade da planta ao corte, como relatado também por Soares Filho et al. (2002).

Os teores de PB nas amostras coletadas em abril, agosto e outubro de 2012 (3,5; 2,3 e 1,5\%, respectivamente) estiveram muito abaixo do valor mínimo de 6\% preconizado por Poppi e McLennan (1995) para que não haja limitação no consumo de MS. O mesmo resultado pôde ser 
observado no mês de julho de 2013, em que o teor de $\mathrm{PB}$ do pasto atingiu $4,0 \%$.

Mesmo com valores de PB aquém daqueles preconizados e que poderiam acarretar redução na digestão delas, devido aos inadequados níveis de nitrogênio para os microrganismos do rúmen, as fêmeas receberam, no período da seca, suplementação proteica com aproximadamente $45,7 \%$ de PB na sua composição final.

Os pesos médios das 98 novilhas ao início e no final da EM e dos períodos secos e chuvosos da primeira EM, bem como os pesos médios das 36 novilhas gestantes na primeira EM (três G, nove GN e 24 NA) com idade média de 26 meses, são apresentados na Tab. 1.

Tabela 1. Pesos corporais de fêmeas bovinas Guzerá (G), 1/2 Guzerá x 1/2 Nelore (GN) e Red Angus x 1/2 Nelore (AN), durante sua primeira e segunda estações de monta (EM)

\begin{tabular}{|c|c|c|c|}
\hline & Peso vivo $(\mathrm{kg})$ & & \\
\hline Épocas das pesagens & $\mathrm{G}$ & GN & $\mathrm{AN}$ \\
\hline Início da $1^{a} \operatorname{EM}(09 / 02 / 2012)$ & $245,33 \pm 8,39 b$ & $256,56 \pm 30,86 b$ & $306,63 \pm 27,34 \mathrm{a}$ \\
\hline Final da $1^{\mathrm{a}} \operatorname{EM}(05 / 05 / 2012)$ & $298,33 \pm 8,50 b$ & $312,22 \pm 28,83 b$ & $364,58 \pm 26,81 \mathrm{a}$ \\
\hline Início da seca $(15 / 06 / 2012)$ & $306,67 \pm 11,02 b$ & $328,50 \pm 31,29 b$ & $371,92 \pm 28,92 \mathrm{a}$ \\
\hline Início das águas $(05 / 10 / 2012)$ & $324,67 \pm 22,81 b$ & $340,44 \pm 33,87 \mathrm{ab}$ & $370,00 \pm 26,32 \mathrm{a}$ \\
\hline Início da $2^{\mathrm{a}} \operatorname{EM}(25 / 01 / 2013)$ & $313,67 \pm 25,01 b$ & $336,50 \pm 45,84 b$ & $399,86 \pm 44,45 \mathrm{a}$ \\
\hline Final da $2^{\circ} \operatorname{EM}(01 / 04 / 2013)$ & $366,33 \pm 4,93 b$ & $389,22 \pm 55,06 \mathrm{ab}$ & $433,79 \pm 37,93 a$ \\
\hline
\end{tabular}

* Médias de peso com letras diferentes, na mesma linha $(\mathrm{P}<0,05)$.

Como observado na Tab. 1, os pesos ao início da segunda estação de monta dos animais das raças G e GN diferiram do encontrado para os animais $\mathrm{AN}$, sendo este superior àqueles $(\mathrm{P}<0,05)$. Esse padrão também é observado na primeira estação de monta (EM) destes animais e no início da primeira seca.

Por ocasião da segunda estação reprodutiva, a produção de MST/ha foi mais elevada, estando acima de $2.500 \mathrm{~kg} / \mathrm{ha}$, e as pastagens apresentaram teores de PB (7,6 a 12,4\%) mais elevados que na primeira EM. Foi observado, no mesmo período, que as primíparas dos grupos $\mathrm{G}$ e GN registraram ganho médio diário (GMD) de $0,74 \pm 0,35$ e $0,74 \pm 0,30$, enquanto as fêmeas da raça AN registraram ganho inferior, de $0,57 \pm 0,36(\mathrm{P}>0,05)$.

As diferenças de peso corporal podem ser explicadas pelas variações genotípicas dos grupos experimentais com superioridade para os animais cruzados, mostrando como a heterose pode influenciar no desenvolvimento ponderal. Nos cruzamentos com Bos taurus indicus, observa-se maior adaptação dos animais a níveis nutricionais limitantes e, consequentemente, mais adequadas respostas produtiva e reprodutiva (Cundiff et al., 1993). Segundo Restle et al. (1999), são expressivos os níveis de heterose nas características de desenvolvimento ponderal, idade à puberdade e desempenho reprodutivo de novilhas.

Segundo Fries (1996), o grau de heterose obtido nos cruzamentos depende de vários fatores, dentre eles a distância genética entre as raças envolvidas, em que quanto maior a distância genética maior a heterose. Embora os dois grupos apresentem heterose devido ao cruzamento de raças distintas, a distância genética entre a raça Red Angus (Bos taurus taurus) e Nelore (Bos taurus indicus) é maior que aquela entre as raças Nelore e Guzerá (Bos taurus indicus).

As novilhas gestantes dos grupos $G$ e $G N$ perderam, respectivamente, $11,0 \mathrm{~kg}$ e $4,0 \mathrm{~kg}$, enquanto as da raça AN ganharam $29,0 \mathrm{~kg}$ no período entre o parto, que corresponde ao início 
das águas, ao início da segunda estação de monta (Tab. 1). Perdas de peso no período pós-parto também foram observadas por Vieira et al. (2005), em que as novilhas apresentaram peso de $436 \pm 30,86 \mathrm{~kg}$ antes do parto e condição corporal ao parto de $4,57 \pm 0,4$. Já no meio da estação de acasalamento, o peso vivo e a condição corporal foram de $360 \pm 31,00 \mathrm{~kg}$ e $2,94 \pm 0,60$.

Ao avaliarem o desempenho reprodutivo de novilhas de corte aos dois anos de idade, Rocha e Lobato (2002) observaram que o grupo racial afetou de forma significativa o peso no início da estação de acasalamento $(\mathrm{P}<0,05)$. Novilhas Hereford apresentaram maior peso $(\mathrm{P}<0,01)$ do que as novilhas cruzadas, que não diferiram entre si $(\mathrm{P}>0,05)$. Novilhas Hereford foram 14,8 e $18,8 \mathrm{~kg}$ mais pesadas, respectivamente, que as $1 / 4$ Nelore e 3/8 Nelore, enquanto os resultados obtidos no presente estudo mostraram a superioridade em ganho de peso de animais cruzados (Bos taurus taurus $x$ Bos taurus indicus), que foram 86,1 e $63,3 \mathrm{~kg}$ mais pesados que os animais zebuínos (Bos taurus indicus) e seus cruzamentos (Bos taurus indicus $x$ Bos taurus indicus) no início do segundo período reprodutivo, respectivamente.

Como mostra a Tab. 2, ocorreu perda de escore de condição corporal nas fêmeas de todos os diferentes grupos genéticos no período entre o pré-parto (início da época das águas) e o início da segunda EM, sendo a perda no grupo $\mathrm{AN}$ (1,99 ponto) maior que nos grupos $G(1,34$ ponto) e GN (1,75 ponto) $(\mathrm{P}<0,05)$, ou seja, apesar de as fêmeas $\mathrm{AN}$ terem maiores pesos corporais no início dos períodos das águas e da seca (Tab. 1), perderam maior condição corporal nesse mesmo intervalo. As perdas de condições corporais observadas podem ser justificadas pela limitação nutricional diretamente relacionada com a composição químico-bromatológica das pastagens de Brachiaria brizantha cv Marandu, pela composição genética dos animais e pela maior necessidade nutricional da matriz com o bezerro ao pé.

Tabela 2. Médias dos escores de condições corporais dos animais experimentais na primeira e segunda estações de monta

\begin{tabular}{|c|c|c|c|}
\hline & \multicolumn{3}{|c|}{ Escore de condição corporal } \\
\hline & Guzerá & $1 / 2$ Guzerá x $1 / 2$ Nelore & Red Angus x $1 / 2$ Nelore \\
\hline Início da $1^{\mathrm{a}} \operatorname{EM}(09 / 02 / 2012)$ & $5,33 \pm 0,58 b$ & $5,33 \pm 0,50 \mathrm{ab}$ & $6,00 \pm 1,00 \mathrm{a}$ \\
\hline Final da $1^{\mathrm{a}} \operatorname{EM}(05 / 05 / 2012)$ & $6,00 \pm 0,00 \mathrm{~b}$ & $5,67 \pm 0,51 \mathrm{ab}$ & $6,75 \pm 0,53 a$ \\
\hline Início da seca (15/06/2012) & $5,00 \pm 0,00 \mathrm{~b}$ & $5,89 \pm 0,60 \mathrm{ab}$ & $6,38 \pm 0,58 \mathrm{a}$ \\
\hline Início das águas (05/10/2012) & $6,67 \pm 0,58 b$ & $7,00 \pm 0,71 b$ & $7,35 \pm 0,49 a$ \\
\hline Início da $2^{\mathrm{a}} \operatorname{EM}(25 / 01 / 2013)$ & $5,33 \pm 0,58 b$ & $5,25 \pm 1,04 b$ & $5,36 \pm 1,09 a$ \\
\hline Final da $2^{\circ} \operatorname{EM}(01 / 04 / 2013)$ & $5,67 \pm 0,58 b$ & $6,00 \pm 1,00 \mathrm{~b}$ & $6,30 \pm 0,76 a$ \\
\hline
\end{tabular}

Médias de escore de condição corporal com letras diferentes, na mesma linha $(\mathrm{P}<0,05)$.

Os escores das condições corporais registrados estão dentro do sugerido por Santos et al. (2009) no período antes do parto (início das águas), apresentando os animais das raças G, GN e AN condições corporais de $6,67 \pm 0,58 ; 7,00 \pm 0,71$ e $7,35 \pm 0,49$, respectivamente. Já em relação ao período pós-parto, esses animais apresentaram escores de condição corporal aquém do indicado pelo mesmo autor, com 5,33 $\pm 0,58 ; 5,25 \pm 1,04$ e $5,36 \pm 1,09$, para $\mathrm{G}, \mathrm{GN}$ a $\mathrm{AN}$, respectivamente.
Na segunda EM, houve diferença $(\mathrm{P}<0,05)$ nos valores encontrados para as taxas de reconcepção, com superioridade três vezes maior para os animais AN (58,33\%) que o registrado para GN $(22,22 \%)$, diferindo dos animais $\mathrm{G}(0 \%)$ (Tab. 3). Animais da raça $\mathrm{G}$ diferiram $(\mathrm{P}<0,05)$ desses grupos, com taxas de concepção de $9,09 \%$ e taxa de reconcepção de $0 \%$. 
Tabela 3. Total de fêmeas gestantes na primeira e segunda estações de monta e taxas de concepção e reconcepção de fêmeas GN e AN

\begin{tabular}{llllllll}
\hline $\begin{array}{l}\text { Grupo } \\
\text { genético }\end{array}$ & $\mathrm{N}$ & $\begin{array}{l}\text { Número de } \\
\text { prenhez } \\
1^{\mathrm{a}} \mathrm{EM}\end{array}$ & $\begin{array}{l}\text { Taxa de } \\
\text { nancepção } \\
(\%)\end{array}$ & $\begin{array}{l}\text { Número de } \\
\text { prenhez na } 2^{\mathrm{a}} \\
\text { EM }\end{array}$ & $\begin{array}{l}\text { Taxa } \\
\text { reconcepção } \\
(\%)\end{array}$ & $\begin{array}{l}\text { Prenhezes } \\
\text { acumuladas }\end{array}$ \\
\hline GN & 32 & 9 & $28,13 \mathrm{~b}$ & 2 & $22,22 \mathrm{a}$ & 11 \\
AN & 32 & 24 & $75,00 \mathrm{a}$ & 14 & $58,333 \mathrm{a}$ & 38 \\
\hline
\end{tabular}

Letras diferentes na mesma coluna diferem $(\mathrm{P}<0,05)$ entre si.

Os resultados referentes às taxas de reconcepção registradas para cada grupo genético mostraram relação com o ganho de peso e o escore corporal dos animais durante todo o período experimental. Constatou-se que, nos meses em que a produção de MST/ha foi relativamente baixa (agosto e outubro do ano de 2012) em relação a todo o período experimental, houve perda de peso e de escore corporal das fêmeas. Esse período, que é compreendido como o período que antecede ao parto e que ocorre na época das secas, também apresentou teores de PB e valores de DIVMS inferiores ao esperado. Mesmo com a utilização de suplementos múltiplos na seca, isso não impediu que os animais perdessem peso, sugerindo que tal suplementação não tenha suprido todos os requisitos nutricionais dos animais nessa fase reprodutiva. Esses fatores podem ter sido decisivos para as taxas reprodutivas encontradas, já que muitos autores confirmam a relação do peso ao pré e pós-parto com a taxa de reconcepção (Almeida et al., 2002; Gottschall et al., 2008).

No período pré-parto (início das águas), as fêmeas do grupo AN apresentaram os maiores pesos corporais e escores das condições corporais, respectivamente, $370,0 \mathrm{~kg}$ e 7,35 . Esses valores só diferiram $(\mathrm{P}<0,05)$ dos encontrados para as fêmeas do grupo $\mathrm{G}$, que, no mesmo período, apresentavam peso vivo médio de $324,0 \mathrm{~kg}$ e escore de condição corporal de 6,67. As taxas de reconcepção obtidas para $\mathrm{AN}$ e GN foram de 58,33 e $22,22 \%$, respectivamente, enquanto para as fêmeas do grupo $\mathrm{G}$ foi de zero.

Mesmo com a manutenção de um escore de condição corporal antes do parto acima de 5 (escala de 1 a 9), como estabelecido por Lobato (2003), isso não foi o suficiente para determinar altas taxas de reconcepção nas fêmeas da raça Guzerá, principalmente por se tratar de novilhas que foram desafiadas aos 14 meses de idade, sem nenhuma seleção prévia para precocidade sexual. Esse resultado sugere, mais uma vez, que o fator genético é determinante no processo reprodutivo, indicando que a heterose seja a possível responsável pela diferença registrada entre animais cruzados em comparação com animais puros e que haja superioridade daqueles sobre estes.

Alves Filho e Restle (1998), ao avaliarem a variação anual de pesos e condições corporais de vacas das raças Charolês $(\mathrm{C})$, Nelore $(\mathrm{N}), 1 / 2 \mathrm{CN}$ e $1 / 2 \mathrm{NC}$, contemporâneas, recém-paridas e que reconceberam, concluíram que as vacas F1 apresentaram estado corporal superior durante todo o experimento em comparação com as vacas puras $(\mathrm{P} \leq 0,01)$, as quais apresentaram escore de condição corporal médio de 2,85, enquanto as cruzadas apresentaram CC de 3,06 (escala de 1 a 5).

As fêmeas dos grupos GN e AN apresentaram, ao início da segunda estação de monta, pesos e escores corporais superiores às fêmeas da raça $\mathrm{G}$, embora sem diferença $(\mathrm{P}<0,05)$ entre $\mathrm{G}$ e GN. Neste estudo, não houve correlação expressiva e significativa entre a taxa de reconcepção e o escore corporal aferido no início da segunda estação reprodutiva $(r=0,12)$, porém registrouse correlação mediana $(r=0,59)$ entre $\mathrm{CC}$ no início da primeira estação de monta e à reconcepção. Assim, animais do grupo AN que apresentaram maiores pesos na primeira estação de monta e que tiveram, ao longo de todo o experimento, escore de condição corporal significativamente $(\mathrm{P}<0,05)$ maior que os da raça $\mathrm{G}$ e GN, obtiveram também as maiores taxas de reconcepção $(58,33 \%)$.

As fêmeas dos grupos G, GN e AN apresentaram prenhez acumulada de 3, 11 e 38, respectivamente. Ao se associarem esses valores com os pesos e escores corporais durante a fase 
experimental, pôde-se perceber que as fêmeas da raça $G$ entraram na primeira e na segunda estação de monta com o peso, respectivamente, abaixo dos $65 \%$ e $85 \%$ do peso adulto, que é recomendado para que haja uma taxa de concepção aceitável (Rovira, 1974). Isso sugere que principalmente as fêmeas da raça (G) deveriam entrar na sua primeira estação de monta com idade mais avançada ou reprodutivamente mais precoces se fosse realizado um manejo adequado, permitindo ganho de peso apropriado até a primeira estação de monta. As fêmeas da raça Guzerá foram produtivamente inviáveis pelo pequeno número de animais que conceberam na primeira EM e que reconceberam. Tais animais poderiam ser usados com a finalidade de seleção de linhagens de animais mais precoces dentro do rebanho.

Apesar de o peso corporal ter demonstrado grande influência na taxa de reconcepção, houve baixa correlação entre o peso no início da primeira $(\mathrm{r}=0,28)$ e da segunda EM $(\mathrm{r}=0,21)$ com a taxa de reconcepção. Para alguns autores, independentemente do ganho, é o peso ao início do período reprodutivo que apresenta papel fundamental em sistemas intensivos de pecuária de corte (Rovira, 1996; Rocha e Lobato, 2002; Pilau e Lobato, 2008).

\section{CONCLUSÃO}

Os maiores pesos corporais ao início da primeira e da segunda estação de monta refletiram em maior condição corporal, maior taxa de concepção e reconcepção para o grupo das matrizes primíparas cruzadas $1 / 2$ Red Angus x 1/2 Nelore, com relação às primíparas das raças $1 / 2$ Guzerá x $1 / 2$ Nelore e Guzerá, demonstrando que a heterose resultante do cruzamento entre raças distintas com maior distância genética (Bos taurus taurus $x$ Bos taurus indicus) proporciona maiores desempenhos produtivo e reprodutivo.

\section{REFERÊNCIA}

ALENCAR, M.M. Perspectivas para o melhoramento genético de bovinos de corte no Brasil. In: SIMPÓSIO SOBRE MELHORAMENTO ANIMAL; REUNIÃO ANUAL DA SOCIEDADE BRASILEIRA DE ZOOTECNIA, 41., 2004, Campo Grande, MS, Anais... Campo Grande: SBZ, 2004. p.358-367.
ALMEIDA, L.S.P.; LOBATO, J.F.P.; SCHENKEL, F.S. Data de desmame e desempenho reprodutivo de vacas de corte. Rev. Bras. Zootec., v.31, p.1223-1229, 2002.

ALVES FILHO, D.C.; RESTLE, J. Variação anual do peso e estado corporal de vacas de diferentes grupos genéticos. 1. Vacas paridas e com prenhez positiva na sequência. Semina Ciênc. Agr., v.19, p.54-59, mar. 1998.

BRAGA, G.J.; PEDREIRA, G.S.; HERLING, V.R. et al. Eficiência de pastejo de capim- marandu submetido a diferentes ofertas de forragem. Pesqui. Agropecu. Bras., v.42, p.1641-1649, 2007.

CUNDIFF, L.; SZABO, F.; GREGORY, K. et al. Breed comparisons from the MARC-ARS-USDA germoplasm evaluation program. In: MEETING OF BEEF IMPROVEMENT FEDERATION, 25., 1993, Asheville. Proceedings... Asheville: [s.n.], 1993. 17p.

DETMANN, E.; SOUZA, M.A.; VALADARES FILHO, S.C. et al. Métodos para análise de alimentos. Visconde Do Rio Branco: Suprema, 2012, 214p.

EUCLIDES, V.P.B.; EUCLIDES FILHO, K.; ARRUDA, Z.J. et al. Desempenho de novilhos em pastagem de Brachiaria decumbens submetidos a diferentes regimes alimentares. Rev. Bras. Zootec., v.27, p.246-254, 1998.

EUCLIDES, V.P.B.; CARDOSO, E.G.; MACEDO, M.C.M. et al. Consumo voluntário de Brachiaria decumbens cv. Basilik e Brachiaria brizantha cv. Marandu sob pastejo. Rev. Bras. Zootec., v.29, supl 2, p.2200-2208, 2000 .

FONSECA, V.O. Redução do período de serviço em vacas de corte . In: CONGRESSO BRASILEIRO DE REPRODUÇÃO ANIMAL, 9., 1991, Belo Horizonte. Anais... Belo Horizonte : CBRA., 1991. v.2, p.1-21.

FLORES, R.S.; EUCLIDES, V.P.B.; ABRÃO, M.P.C. et al. Desempenho animal, produção de forragem e características estruturais dos capins marandu e xaraés submetidos a intensidades de pastejo. Rev. Bras. Zootec., v.37, p.1355-1365, 2008.

FRIES, L.A. Calculando e decompondo heterozigoses. In: SIMPÓSIO NACIONAL DE MELHORAMENTO ANIMAL, 1., 1996, Ribeirão Preto. Anais ... Ribeirão Preto: SBMA, 1996. p.252-254.

GOTTSCHALL, C.; FERREIRA, E.; CANELLAS, L. et al. Perdas reprodutivas e reconcepção em bovinos de corte segundo a idade ao acasalamento. Arq. Bras. Med. Vet. Zootec., v.60, p.414-418, 2008.

HIGHT, G.K. The effects of the under nutrition in late pregnancy on beef cattle production. New Zeal. Agric. Res., v.9, p.479-490, 1966. 
LOBATO, J.F.P. A "vaca ideal" e o seu manejo em sistemas de produção de ciclo curto. In: SIMPÓSIO DA CARNE BOVINA: DA PRODUÇÃO AO MERCADO CONSUMIDOR, 1., 2003, São Borja. Anais... Porto Alegre: Universidade Federal do Rio Grande do Sul, 2003. p.9-43.

LOWMAN, B.G.N.; SCOTT, N.A.; SOMERVILLE, S.H. Condition scoring of cattle. Edinburgh: The Edinburgh School of Agriculture, 1976. 5p. (East of Scotland College of Agriculture Bulletin, 6).

McMENIMAN, N.P. Methods of estimating intake of grazing animals. In: REUNIÃO ANUAL DA SOCIEDADE BRASILEIRA DE ZOOTECNIA, 34., Juiz de fora, 1997. Anais... Juiz de Fora: Sociedade Brasileira de Zootecnia, 1997. p.131-168.

PAIM, T.P.; BATTISTELLI, J.V.F.; SOUZA, M.D. et al. Precocidade sexual de novilhas cruzadas. In: JORNADA CIENTÍFICA DA EMBRAPA GADO DE CORTE, 6., 2010, Campo Grande, MS. Anais... Campo Grande, MS: Embrapa Gado de Corte, 2010, CD-ROM.

PEREIRA NETO, O.A.; LOBATO, J.F.P. Efeitos da ordem de utilização de pastagens nativas melhoradas no desenvolvimento e comportamento reprodutivo de novilhas de corte. Rev. Bras. Zootec., v.27, p.60-65, 1998.

PILAU, A.; LOBATO, J.F.P. Manejo de novilhas prenhes aos 13/15 meses de idade em sistemas a pasto. Rev. Bras. Zootec., v.37, p.1271-1279, 2008.

RESTLE, J., POLLI, V.A., SENNA, D.B. Efeito de grupo genético e heterose sobre a idade e peso a puberdade e sobre o desempenho reprodutivo de novilhas de corte. Pesqui. Agropecu. Bras., v.34, p.701-707, 1999.

RICHARD, M.W.; SPITZER, J.C.; WARNER, M.B. Effect of varying levels of pospartum nutrition and body condition at calving on subsequent reproductive performance in beef cattle. J. Anim. Sci., v.62, p.300306, 1986.
ROCHA, M.G.; LOBATO, J.F.P. Avaliação do desempenho reprodutivo de novilhas de corte primíparas aos dois anos de idade. Rev. Bras. Zootec., v.31, supl., p.1388-1395, 2002.

ROVIRA, J. Reproducción y manejo de los rodeos de cria. Montevideo: Hemisferio Sur, 1974. p.293.

ROVIRA, J.M. Manejo nutritivo de los rodeos de cría en pastoreo. Montevideo: Hemisferio Sur, 1996. p.288.

SANTOS, S.A.; ABREU, U.G.P.; SOUZA, G.S. et al. Condição corporal, variação de peso e desempenho reprodutivo de vacas de cria em pastagem nativa no Pantanal. Rev. Bras. Zootec., v.38, p.354-360, 2009.

SILVA, R.R.; PRADO, I.N.; PINTO de CARVALHO, G.G. et al. Níveis de suplementação na terminação de novilhos Nelore em pastagens: aspectos econômicos. Rev. Bras. Zootec., v.39, p.2091-2097, 2010.

SNEDECOR, G.W.; COCHRAN, W.G. Statistical methods. Iowa: The Iowa State University Press, 1980. $507 \mathrm{p}$.

SOARES FILHO, C.V.; RODRIGUES, L.R.A.; PERRI, S.H.V. et al. Produção e valor nutritivo de dez gramíneas forrageiras na região noroeste do estado de São Paulo. Acta Sci., v.24, p.1377-1384, 2002.

STATISTICAL ANALYSIS SYSTEM - SAS. SAS users guide: statistics. Cary: 2004. (CD-ROM).

VIEIRA, A.; LOBATO, J.F.P.; TORRES JUNIOR, R.A.A. et al. Fatores determinantes do desempenho reprodutivo de vacas Nelore na região dos cerrados do Brasil Central. Rev. Bras. Zootec., v.34, supl., p.24082416, 2005. 